ORIGINAL

\title{
Efecto del plasma seminal sobre el estado redox del semen equino criopreservado
}

\section{Effect of seminal plasma on the redox state of cryopreserved stallion semen}

\author{
Edison Pizarro L, ${ }^{1}$ Zoot, Giovanni Restrepo B, ${ }^{1 *}$ M.Sc, José Echeverry Z, ${ }^{2}$ Ph.D, \\ Benjamín Rojano, ${ }^{2}$ Ph.D.
}

\begin{abstract}
${ }^{1}$ Politécnico Colombiano Jaime Isaza Cadavid, Carrera 48 No 7-151, Medellín, Colombia. ${ }^{2}$ Universidad Nacional de Colombia Sede Medellín, Calle 59A No 63-20, Medellín, Colombia. Correspondencia: grestrepo@elpoli.edu.co
\end{abstract}

Recibido: Septiembre de 2012; Aceptado: Febrero de 2013.

\section{RESUMEN}

Objetivo. Determinar el efecto del plasma seminal sobre la generación de especies reactivas de oxígeno (ERO) y la peroxidación lipídica de semen equino criopreservado y su asociación con parámetros de calidad seminal. Materiales y métodos. El semen de cinco caballos de la raza criollo colombiano (dos eyaculados cada uno), fue criopreservado mediante un protocolo de congelación rápida, empleando un diluyente leche-yema de huevo, suplementado con $0 \%, 10 \%$ y $20 \%$ de plasma seminal equino. En muestras de semen fresco y criopreservado se evaluó la generación de ERO y la peroxidación lipídica por espectrofluorimetría, y los parámetros de calidad seminal de movilidad progresiva, vitalidad e integridad de membrana, mediante microscopia de contraste de fase. Para el análisis estadístico se ajustaron modelos mixtos y se realizaron análisis de regresión y correlación. Resultados. Se hallaron promedios post-descongelación de movilidad progresiva, vitalidad e integridad de membrana de $37.8 \% \pm 20.2$, $50.6 \% \pm 14.6$ y $37.8 \% \pm 15.5$, respectivamente. Para el semen fresco y criopreservado suplementado con $0 \%, 10 \%$ y $20 \%$ de plasma seminal, los promedios de producción de ERO (URF) fueron de $13.34 \pm 10.7,16.15 \pm 13.5,17.32 \pm 16$ y $22.98 \pm 19.4$, respectivamente; mostrando un incremento estadísticamente significativo $(\mathrm{p} \leq 0.05)$ en la producción de ERO por efecto de la criopreservación y la suplementación con plasma seminal. Los promedios de peroxidación lipídica (nmolMDA/ml) para estos mismos tratamientos, fueron de $0.41 \pm 0.25,0.72 \pm 0.37,0.51 \pm 0.29$ y $0.47 \pm 0.26$, respectivamente; mostrando una reducción significativa $(\mathrm{p} \leq 0.05)$ de la peroxidación lipídica del semen suplementado con $10 \%$ y $20 \%$ de plasma seminal, respecto al semen no suplementado (0\%). Conclusiones. El plasma seminal reduce la peroxidación lipídica del semen equino criopreservado.

Palabras clave: Antioxidantes, criopreservación, especies reactivas de oxígeno (Fuente: MeSH). 


\begin{abstract}
Objective. Determine the effect of seminal plasma on the generation of reactive oxygen species (ROS) and lipid peroxidation of cryopreserved stallion semen, and its association with semen quality parameters. Materials and methods. The semen of five stallions of Colombian creole breed (two ejaculates each) was cryopreserved by a rapid freezing protocol, using a milk-egg yolk extender supplemented with $0 \%, 10 \%$ and $20 \%$ of equine seminal plasma. The samples of fresh and cryopreserved semen were evaluated for ROS generation and lipid peroxidation by spectrofluorimetry, and semen quality parameters of progressive motility, vitality and membrane integrity using phase contrast microscopy. Mixed models were adjusted for statistical, regression, and correlation analysis. Results. Post-thaw averages of progressive motility, vitality and integrity of membrane of $37.8 \% \pm$ $20.2,50.6 \% \pm 14.6$ and $37.8 \pm 15.5 \%$, respectively were found. For fresh and cryopreserved semen supplemented with $0 \%, 10 \%$ and $20 \%$ of seminal plasma, the averages of ROS production (RFU) were $13.34 \pm 10.7,16.15 \pm 13.5,17.32 \pm 16$ and $22.98 \pm 19.4$, respectively; showing a statistically significant increase $(p \leq 0.05)$ of ROS production by effect of cryopreservation and seminal plasma supplementation. The averages of lipid peroxidation $(\mathrm{nmolMDA} / \mathrm{ml}$ ) for these same treatments were $0.41 \pm 0.25,0.72 \pm 0.37,0.51 \pm 0.29$ and $0.47 \pm 0.26$, respectively; showing a significant decrease $(p \leq 0.05)$ of lipid peroxidation of semen supplemented with $10 \%$ and $20 \%$ of seminal plasma compared to unsupplemented semen $(0 \%)$. Conclusions. Seminal plasma reduces lipid peroxidation of stallion cryopreserved semen.
\end{abstract}

Key words: Antioxidants, cryopreservation, reactive oxygen species (Fuente: MeSH).

\section{INTRODUCCIÓN}

El uso generalizado y efectivo de semen equino criopreservado es aún limitado, principalmente por su asociación con la baja fertilidad. Como es conocido, los espermatozoides de esta especie son extremadamente sensibles a las alteraciones celulares generadas por la congelación (1). El mejoramiento de los procedimientos de criopreservación de semen equino pueden derivarse en un uso más eficiente de biotecnologías reproductivas como la inseminación artificial, la transferencia de embriones y la fecundación in vitro. Diferentes técnicas y crioprotectores han sido evaluados $(2,3)$. Sin embargo, han persistido alteraciones marcadas sobre la movilidad, la viabilidad y la morfología de los espermatozoides equinos $(4,5)$, las cuales a la vez se han atribuido al estrés térmico, al estrés osmótico y principalmente al estrés oxidativo involucrado en los procesos de criopreservación $(1,6)$. El peróxido de hidrógeno $\left(\mathrm{H}_{2} \mathrm{O}_{2}\right)$ es la especie reactiva de oxígeno (ERO) más comprendida en el daño de los espermatozoides equinos, siendo generada por la acción de la superóxidodismutasa (SOD) sobre el anión superóxido, el cual es el principal radical libre producido por el espermatozoide equino (7). Cuando las ERO (especies reactivas de oxígeno) son generadas en exceso, se presenta una consecuente disminución de la fertilidad, principalmente como resultado de la peroxidación de los lípidos de membrana, lo cual es promovido por el alto contenido de ácidos grasos poliinsaturados de los espermatozoides equinos $(8,9)$.
Adicionalmente, se ha planteado que los procesos de congelación de semen, conducen a la perdida de la actividad de defensa antioxidante, por el daño estructural del citoesqueleto y de las enzimas antioxidantes $(10,11)$. Para contrarrestar, anular o minimizar los efectos de las ERO sobre los espermatozoides, se ha suplementado el semen equino con una variedad de sistemas antioxidantes, entre los cuales se encuentran SOD, catalasa (CAT), glutatión reducido, ácido ascórbico y a-tocopherol $(12,13)$. La remoción del plasma seminal durante el procesamiento del semen para su criopreservación, también favorece el estrés oxidativo del semen equino, toda vez que la mayoría de su capacidad antioxidante se encuentra allí (1). El plasma seminal equino posee actividades considerables de SOD y de glutatión-peroxidasa (GPx) (14), y otras moléculas presentes en el plasma seminal, como vitamina $C$, vitamina $E$, urato, albumina, taurina, hipotaurina, piruvato y lactato, podrían actuar como antioxidantes $(15,16)$.

El objetivo de esta investigación fue evaluar el efecto de la suplementación con plasma seminal, en un diluyente para congelación, sobre el estado redox (producción de ERO y peroxidación lipídica) y la fertilidad potencial de semen equino criopreservado. 


\section{MATERIALES Y MÉTODOS}

Recolección de material de investigación. Cinco caballos de la raza criollo colombiano, localizados en un predio (latitud $6^{\circ} 23^{\prime} 41.50^{\prime \prime} \mathrm{N}$, longitud $\left.75^{\circ} 25^{\prime} 27.95^{\prime \prime} \mathrm{O}\right)$ del municipio de Girardota, Antioquia (Colombia), fueron utilizados para la recolección de dos eyaculados por animal, con una frecuencia semanal. Los ejemplares estuvieron entre los 2 y 8 años de edad, todos en un régimen de mínimo una colecta semanal, con fertilidad comprobada con crías nacidas vivas y con una condición corporal de 6 a 7 (escala entre 1 y 9 , siendo 1 extremadamente delgado y 9 extremadamente obeso). Fueron sometidos a condiciones iguales de manejo en estabulación, a una alimentación a base de pasto estrella henificado (Cynodon nlemfuensis) suministrado a voluntad, $4 \mathrm{~kg}$ diarios de concentrado distribuidos en 8 raciones, suplementación multi-vitamínica semanal, vermifugación semestral y vacunación anual contra el virus de la Encefalitis equina venezolana.

Recolección del semen. Se realizó por el método de vagina artificial, utilizando una vagina modelo Missouri lubricada con gel no espermicida. Una yegua fue empleada para la colecta, y la fracción en gel del eyaculado fue removida por filtración. Posteriormente, el semen recién recolectado fue diluido en proporción $1: 1$ en diluyente EquiPro $\AA$ (Minitube) a $37^{\circ} \mathrm{C}$. De cada eyaculado, entre 5 y $10 \mathrm{ml}$ de semen (sin diluir) fueron sometidos a centrifugación a $3000 \mathrm{rpm}$ durante 15 minutos; el plasma seminal fue recuperado con el fin de emplearlo posteriormente como suplemento para la criopreservación, mientras el precipitado de células espermáticas fue descartado. Tanto el plasma seminal como el semen diluido fueron transportados en refrigeración $\left(5^{\circ} \mathrm{C}\right)$ hacia el laboratorio, dentro de los 30 minutos siguientes.

Evaluación seminal. Utilizando un microscopio de contraste de fase Eclipse E200 (Nikon Inc.), se determinó la movilidad espermática promediando los porcentajes de movilidad progresiva de cinco diferentes campos de observación (400X). La concentración del semen fue determinada a partir de una gota de semen fresco, mediante un fotómetro Spermacue $₫$ (Minitube). La morfología y la vitalidad espermática se determinaron mediante la técnica modificada de eosinanigrosina (17). Sobre un portaobjetos temperado a $37^{\circ} \mathrm{C}$, se depositó una gota de semen, y a su lado una gota de colorante eosina-nigrosina (Sigma-Aldrich 861006 y N4763) a $37^{\circ} \mathrm{C}$; ambas gotas se mezclaron durante 30 a 60 segundos, y se realizó un extendido y la fijación del mismo con calor sobre una platina térmica. Se observó la morfología individual de 200 espermatozoides al microscopio (1000X), y se clasificaron como morfológicamente normales o anormales (18). Se clasificaron como vivos aquellos espermatozoides que no incorporaron el colorante (espermatozoides blancos) y como muertos aquellos que incorporaron el colorante (espermatozoides rosados). Se establecieron porcentajes de espermatozoides normales (\% de morfología normal), así como de espermatozoides vivos ( $\%$ de vitalidad).

Se evaluó la integridad de la membrana plasmática de los espermatozoides por la metodología de la prueba hipoosmótica (HOS) de acuerdo a lo reportado por Neild et al (19), para la cual se tomaron $100 \mu$ lde semen y se adicionaron a un tubo con $500 \mu \mathrm{l}$ de una solución hipoosmótica de sacarosa 5.4\% (100 mosmol/l). Esta mezcla fue incubada a $38.5^{\circ} \mathrm{C}$ por 30 minutos y luego se evaluó el hinchamiento espermático de 100 células en mínimo 5 campos diferentes (400X), utilizando un microscopio de contraste de fase Eclipse E200 (Nikon Inc.). Se determinó el porcentaje de células reaccionadas a partir de 100 espermatozoides contados. Como criterio de selección, solo fueron procesados los eyaculados con parámetros mínimos de $70 \%$ de movilidad progresiva, 100 millones de espermatozoides por $\mathrm{ml}$, y $70 \%$ de espermatozoides morfológicamente normales $(20,21)$.

Criopreservación del semen. La criopreservación del semen se realizó mediante un protocolo modificado de congelación rápida de Bustamante et al (22). El semen diluido fue centrifugado por 10 minutos a $1500 \mathrm{rpm}, \mathrm{y}$ el $90 \%$ del sobrenadante fue descartado. El precipitado fue suspendido en un diluyente EquiPro ${ }^{\circledR}$ suplementado con $4 \%(\mathrm{v} / \mathrm{v})$ de yema de huevo (3) y $5 \%$ de $N, N$ dimetilformamida (Sigma-Aldrich), en cantidad suficiente para una concentración final de $100 \mathrm{x}$ $10^{6}$ espermatozoides por $\mathrm{ml}$. El semen diluido fue dividido en tres alícuotas para su suplementación con $0,10 \circ 20 \%$ de plasma seminal equino (calculados como \% de volumen de diluyente / volumen de plasma seminal). Posteriormente, el semen fue mantenido en refrigeración a $5^{\circ} \mathrm{C}$ por 15 minutos, y luego fue empacado en pajillas de $0.5 \mathrm{ml}$. Las pajillas fueron sometidas a vapores de nitrógeno líquido por 15 minutos; para finalmente ser almacenadas en nitrógeno líquido de acuerdo a un protocolo modificado a partir del reportado por Medeiros et al (23). Después de dos semanas de almacenamiento las pajillas de semen fueron descongeladas en un baño de agua a $37^{\circ} \mathrm{C}$ por 1 minuto, y posteriormente fue evaluada la movilidad progresiva, la vitalidad y la integridad de la membrana plasmática, por las metodologías previamente descritas. 
Determinación del estado redox. La determinación del estado redox se realizó mediante la medición de la producción de ERO y de la peroxidación lipídica tanto en semen fresco como descongelado para los diferentes tratamientos. La detección de especies reactivas se realizó por la metodología de la fluoresceína diacetato -FDA- (24), donde cada muestra contenía $30 \mu \mathrm{l}$ de $40 \mathrm{mM}$ FDA (Intervet), $240 \mu \mathrm{l}$ de solución buffer ( $\mathrm{pH} 7.4)$, y $30 \mu \mathrm{l}$ de semen. Se emplearon condiciones controladas de temperatura a $37^{\circ} \mathrm{C}, \mathrm{pH} 7.4$, y como referencia el antioxidante Trolox $\AA$ (Merck). Las lecturas se realizaron mediante un espectrofluorímetro LS 55 (Perkin Elmer), a una $\lambda$ de excitación $490 \mathrm{~nm}$ y slit de excitación 10 , y una $\lambda$ de emisión 530 nm y slit de emisión 15 . Los resultados fueron expresados como unidades relativas de fluorescencia (URF).

La evaluación de la peroxidación lipídica se realizó por la metodología modificada de las sustancias reactivas al ácido tiobarbitúrico-TBARS- (24); para lo cual $50 \mu \mathrm{L}$ de semen fueron incubados por 1 hora a $37^{\circ} \mathrm{C}$, y luego enfriados en agua con hielo durante 10 minutos. Posteriormente, se adicionaron $40 \mu \mathrm{l}$ de ácido tricloroacético (TCA, Sigma-Aldrich) al 15\%, y $80 \mu \mathrm{l}$ de ácido tiobarbitúrico (TBA, Sigma-Aldrich) al $0.67 \%$ $(\mathrm{p} / \mathrm{v})$, la mezcla fue agitada e incubada por 20 minutos a $90^{\circ} \mathrm{C}$, y luego enfriada en agua con hielo durante 10 minutos. Se adicionaron $300 \mu \mathrm{l}$ de butanol (Sigma-Aldrich), para luego agitar cada muestra por inversión durante 1 min. Finalmente se tomaron $300 \mu$ de la capa superior de la muestra para servirla en una placa múltipozo. El complejo fluorescente formado fue leído en un espectrofluorímetro LS 55 (Perkin Elmer) a una $\lambda$ de excitación $500 \mathrm{~nm}$ y slit de excitación 5 , y $\lambda$ de emisión $550 \mathrm{~nm}$ y un slit de emisión 15. Los resultados fueron expresados como equivalentes de malonaldehido (nmolMDA/ $\mathrm{ml}$ de muestra).

Análisis estadístico. Para la evaluación de las ERO se efectuaron lecturas por cuadriplicado para cada eyaculado y tratamiento, cada 60 segundos durante 80 minutos. Para determinar el efecto de las fuentes de variación se ajustó un modelo mixto de medidas repetidas en el tiempo. El modelo empleado fue:

$\mathrm{Y}_{i j k l}=\mu+\mathrm{T}_{i}+\mathrm{Tto}_{j}+\mathrm{Eq}(\mathrm{Ey})_{\mathrm{k}}+\mathrm{e}_{i j k l,}$ donde:

$Y_{i j k l}^{i j k l}$ : Producción de ERO en el semen.

$\mu$ : $\quad$ Media común a todos los tratamientos.

$\mathrm{T}_{i}$ : $\quad$ Efecto fijo del tiempo $(i) .(i=1 \ldots 81)$.

Tto $_{j}$ : $\quad$ Efecto fijo del tratamiento $(j)$. ( $j=$ fresco, 0, 10, 20\%).

$\mathrm{Eq}(\mathrm{Ey})_{\mathrm{k}}$ : Efecto aleatorio del eyaculado anidado dentro del equino $(\mathrm{k})$.

$\mathrm{e}_{\mathrm{ijkl}}$ : $\quad$ Error aleatorio.
Un modelo de regresión lineal múltiple fue ajustado independientemente para cada uno de los tratamientos, para determinar el efecto del tiempo sobre la producción de ERO, en cada uno. Posteriormente fueron comparados los coeficientes de regresión obtenidos y el ajuste de los modelos utilizados. El modelo de regresión fue:

$Y=\beta_{0}+\beta_{1} X_{1}+\beta_{2} X_{2}+\beta_{3} X_{3}$

donde:

Y: $\quad$ Producción de ERO en el semen

$\beta_{0}: \quad$ Intercepto

$\beta_{1}$ : $\quad$ Efecto del tiempo en la producción de ERO

$X_{1}$ : $\quad$ Tiempo (minutos)

$\beta_{2}$ : $\quad$ Efecto de la edad en la producción de ERO

$\mathrm{X}_{2}$ : $\quad$ Edad (meses)

$\beta_{3}$ : $\quad$ Efecto de la movilidad progresiva en la producción de ERO

$\mathrm{X}_{3}$ : $\quad$ Movilidad progresiva (\%)

Para la evaluación de la peroxidación lipídica (TBARS) se ajustó un modelo mixto completamente aleatorizado con cuatro repeticiones por tratamiento. El modelo empleado fue:

$$
\mathrm{Y}_{i j k l m n}=\mu+\mathrm{Tto}_{i}+\mathrm{R}_{j}+\mathrm{Mov}_{k}+\mathrm{ER}+\mathrm{Eq}(\mathrm{Ey})_{m}
$$

donde:

$Y_{i j k l m n}$ : Peroxidación lipídica en el semen

$\mu$ : Media común a todos los tratamientos.

Tto: $\quad$ Efecto fijo del tratamiento $(i)$. ( $i=$ Fresco, 0, 10, 20\%).

$\mathrm{R}_{j}$ : $\quad$ Efecto fijo de la repetición (pajilla) $(j)$. $(j=1 \ldots 4)$.

Mov $_{k}$ : Efecto fijo de la covariable movilidad progresiva $(k) .(k=0 \ldots 100)$.

ER: $\quad$ Efecto fijo de la covariable velocidad de producción de ERO $(I) .(I=0 \ldots 100)$.

$\mathrm{Eq}(\mathrm{Ey})_{m}$ : Efecto aleatorio del eyaculado anidado dentro del equino $(m)$.

$\mathrm{e}_{\mathrm{ijklmn}}$ : Error aleatorio.

La velocidad de producción de ERO (URF/ minuto) en el semen fresco y el semen congelado para cada tratamiento, se halló mediante el cálculo de la pendiente $(\mathrm{m})$. Para determinar la asociación entre las variables se realizó un análisis de correlación de Pearson de acuerdo con la expresión: $r(X, Y)=\operatorname{cov}(X, Y) / \sigma_{x} \sigma_{y}$. Para la comparación de las medias ajustadas entre los tratamientos, tanto para la producción de ERO como para la peroxidación lipídica, se realizó una prueba de Tukey. Los resultados descriptivos fueron expresados como la media \pm la desviación estándar. Todos los análisis fueron desarrollados mediante el programa SAS para Windows ${ }^{\circledR}$, versión 9.2, SAS Institute Inc. 


\section{RESULTADOS}

Un total de 10 muestras de semen fresco (2 eyaculados por animal) fueron evaluadas $y$ procesadas bajo los diferentes tratamientos. El promedio para los parámetros espermáticos del semen fresco fueron: movilidad progresiva $77.5 \% \pm 10.6$, vitalidad $79.9 \% \pm 8.74$, morfología normal $83.6 \% \pm 5.78$, e integridad de membrana (HOS) $70.4 \% \pm 15.5$. Para el semen criopreservado, se hallaron promedios postdescongelación de movilidad progresiva, vitalidad e integridad de membrana de $37.8 \pm 20.2$, $50.6 \pm 14.6$ y $37.8 \pm 15.5$, respectivamente.

A partir de un total de 1769 registros para la variable producción de ERO, se encontró un promedio de 18.92 URF \pm 15.7. En el modelo mixto ajustado para esta variable, fueron significativos $(p \leq 0.05)$ los efectos fijos de tiempo y tratamiento. Las medias ajustadas de producción de ERO se presentan en la tabla 1. El coeficiente de determinación $\left(R^{2}\right)$ del modelo para ERO fue de 0.77 . Los coeficientes de regresión $(p \leq 0.05)$ hallados para la producción de ERO en el semen bajo los diferentes tratamientos, se describen en la tabla 2.

Tabla 1. Resultados de producción de ERO y peroxidación lipídica en el semen equino.

\begin{tabular}{lcc}
\hline TRATAMIENTO & $\begin{array}{c}\text { Producción ERO } \\
\text { (URF) }\end{array}$ & $\begin{array}{c}\text { Peroxidación } \\
\text { lipídica } \\
\text { (nmMDA/mI) }\end{array}$ \\
\hline Fresco & $13.34 \pm 10.7^{\mathrm{a}}$ & $0.41 \pm 0.25^{\mathrm{a}}$ \\
Plasma 0\% & $16.15 \pm 13.5^{\mathrm{b}}$ & $0.72 \pm 0.37^{\mathrm{b}}$ \\
Plasma 10\% & $17.32 \pm 16.0^{\mathrm{c}}$ & $0.51 \pm 0.29^{\mathrm{c}}$ \\
Plasma 20\% & $22.98 \pm 19.4^{\mathrm{d}}$ & $0.47 \pm 0.26^{\mathrm{c}}$ \\
\hline Letras diferentes indican diferencia estadística significativa $(\mathrm{p} \leq 0.05)$.
\end{tabular}

Tabla 2. Coeficientes de regresión para la producción de ERO en el semen equino fresco y descongelado para los diferentes tratamientos.

\begin{tabular}{lcccc}
\hline TRATAMIENTO & $\boldsymbol{\beta}_{\mathbf{0}}$ (tiempo) & $\boldsymbol{\beta}_{\mathbf{1}}$ (edad) & $\begin{array}{c}\boldsymbol{\beta}_{\mathbf{2}} \\
\text { (movilidad) }\end{array}$ & $\mathbf{R}^{\mathbf{2}}$ \\
\hline Fresco & 0.006 & 0.02 & -0.16 & 0.82 \\
Plasma 0\% & 0.006 & 0.16 & -0.33 & 0.64 \\
Plasma 10\% & 0.008 & 0.01 & -0.58 & 0.84 \\
Plasma 20\% & 0.01 & -0.03 & -0.46 & 0.85 \\
\hline
\end{tabular}

A partir de 122 registros se encontró un promedio para la variable peroxidación lipídica de 0.61 $\mathrm{nmMDA} / \mathrm{ml} \pm 0.32$, mientras los promedios para las covariables incluidas en el modelo, movilidad progresiva y velocidad de producción de ERO fueron de $37.8 \% \pm 20.2$ y $0.0085 \mathrm{URF} / \mathrm{min} \pm$ 0.004 , respectivamente. En el modelo mixto ajustado para esta variable, fue significativo $(p \leq 0.05)$ el efecto fijo del tratamiento, mientras no fueron significativos los efectos fijos repetición, ERO y movilidad progresiva. Se encontró un incremento estadísticamente significativo $(p \leq 0.05)$ de la peroxidación lipídica del semen criopreservado $(0 \%, 10 \%$ y $20 \%$ de plasma seminal), respecto al semen fresco; al igual que una menor peroxidación $(p \leq 0.05)$ del semen suplementado con $10 \%$ (v/v) y $20 \%$ $(\mathrm{v} / \mathrm{v})$ de plasma seminal, respecto al semen no suplementado (Tabla 1 ). El coeficiente de determinación $\left(\mathrm{R}^{2}\right)$ del modelo para peroxidación lipídica fue de 0.74 . Los resultados de los análisis de correlación se presentan en la tabla 3.

Tabla 3. Matriz de coeficientes de correlación (Pearson) entre los parámetros de fertilidad y del estado redox de semen equino criopreservado.

\begin{tabular}{lccccc}
\hline VARIABLE & Peroxidación & ERO & Movilidad & Vitalidad & HOS \\
\hline Peroxidación & & 0.35 & -0.30 & -0.28 & -0.21 \\
ERO & 0.35 & & -0.32 & -0.34 & -0.30 \\
Movilidad & -0.30 & -0.32 & & 0.82 & 0.71 \\
Vitalidad & -0.28 & -0.34 & 0.82 & & 0.70 \\
HOS & -0.21 & -0.30 & 0.71 & 0.70 & \\
\hline
\end{tabular}

\section{DISCUSIÓN}

La criopreservación de semen equino ha sido permanentemente asociada con la baja fertilidad, por el alto impacto de las alteraciones que genera sobre los espermatozoides $(1,25,26)$. Los resultados de esta investigación mostraron una notoria disminución en la movilidad progresiva, la vitalidad y la integridad de membrana en el semen criopreservado respecto al semen fresco.

Tradicionalmente se ha realizado la extracción del plasma seminal en los procesos de criopreservación de semen equino (27), toda vez, que se ha observado que cuando los espermatozoides están expuestos al plasma seminal, son incapaces de sufrir reacción acrosómica y completar la fecundación (28). Sin embargo, se reporta que la remoción del plasma seminal durante la criopreservación favorece el estrés oxidativo del semen equino, dado que la mayoría de su capacidad antioxidante se encuentra allí (1); de manera que las técnicas estándar de criopreservación producen un descenso en la actividad enzimática (21). Katila et al (29), encontraron que puede lograrse un mayor porcentaje de espermatozoides equinos móviles después de la criopreservación, cuando se conserva un $20 \%$ del plasma seminal. También se ha observado un efecto inmunosupresor del plasma seminal, al reducir la unión de los espermatozoides a los polimorfonucleares neutrófilos en el útero después de la inseminación artificial, favoreciendo el proceso de fecundación (30).

Se desconocen en gran medida los factores del plasma seminal relacionados con la fertilidad. 
Recientemente, proteínas del plasma seminal como la proteína secretora rica en cisteína (CRISP3) ha sido asociada positivamente con la tasa de concepción, y es sugerida como un marcador de fertilidad (31). Pessanha et al (32), encontraron que la adición de diferentes concentraciones de proteínas de plasma en el semen equino durante la criopreservación, no mejora los parámetros espermáticos.

El estrés oxidativo ha sido ampliamente relacionado con la reducción de la fertilidad del semen equino (7), por lo cual conocer el aporte del plasma seminal al estado redox del semen destinado a la criopreservación, puede dilucidar sus aportes en la criotolerancia de los espermatozoides. Los resultados de este estudio mostraron como la criopreservación produce un incremento significativo en la producción de ERO en el semen criopreservado, y a la vez como el plasma seminal incrementa aún más dicha producción de forma proporcional cuando se suplementa con 10 y $20 \%$.

Adicionalmente, en el análisis del modelo ajustado para la producción de ERO fue significativo el efecto del tiempo. De acuerdo con los coeficientes de regresión hallados por tratamiento (tabla 2), se produjo un incremento en la velocidad de producción de ERO entre 0.006 URF/min y $0.01 \mathrm{URF} / \mathrm{min}$, lo que demuestra la continua producción de ERO en el semen, produciéndose una acumulación de las mismas que explicaría en parte las alteraciones de los espermatozoides, resultado del estrés oxidativo. La producción de ERO está ligada a la actividad metabólica de los espermatozoides, y depende en gran medida de la disponibilidad de moléculas energéticas, como las presentes en el plasma seminal, así como puede verse afectada por procesos como la centrifugación y la criopreservación (33).

Igualmente se observó un incremento en las ERO por efecto del aumento en edad del equino, probablemente por factores asociados al envejecimiento y a su conocida relación con el estrés oxidativo. Se ha descrito que los espermatozoides provenientes de los animales mas viejos, producen más radicales libres que los provenientes de animales jóvenes, al igual que poseen una reducida actividad antioxidante enzimática (34). También se encontró una reducción de las ERO a causa del aumento en la movilidad progresiva de los espermatozoides (Tabla 2), posiblemente relacionado con una menor presencia de células apoptóticas en el semen, las cuales son conocidas por generar altas cantidades de especies reactivas (35).
Otro factor asociado con la pérdida de la movilidad, viabilidad, y capacidad para la fecundación de los espermatozoides, es el incremento en la peroxidación de los lípidos después de la criopreservación. Como consecuencia de la peroxidación, la membrana plasmática pierde ácidos grasos poliinsaturados con una producción asociada de hidroperóxidos lipídicos, y radicales alcoxilo y peroxilo; los cuales promueven una reacción en cadena de peroxidación lipídica y en última instancia conducen a la producción de aldehídos citotóxicos como malondialdehido y 4-hidroxinonenol (36). Esta situación desencadena alteraciones a nivel de la membrana plasmática, acrosomal y mitocondrial, provocando una reducción en su fluidez e integridad $(9,25)$. De acuerdo a los resultados obtenidos, la peroxidación lipídica presentó un incremento significativo en el semen equino criopreservado ( $0 \%$ plasma seminal), respecto al semen fresco. Este efecto ha sido evidenciado previamente en el semen de esta especie, siendo atribuido al estrés oxidativo del proceso, conjugado con el alto contenido de ácidos grasos poliinsaturados en las membranas de los espermatozoides (25).

La adición del plasma seminal al 10\% (v/v) y al $20 \%$ en la solución de congelación, permitió controlar los procesos de peroxidación lipídica en el semen equino criopreservado, en niveles cercanos a la peroxidación del semen fresco. Este efecto podría atribuirse a la presencia de moléculas con función antioxidante en el plasma seminal. Villa et al (37), encontraron actividades de glutatión-peroxidasa (GPX) de $0.55 \pm 0.05$ $\mathrm{U} / \mathrm{ml}$ y de SOD de $86 \pm 7.1 \mathrm{U} / \mathrm{ml}$ en el plasma seminal de caballos de la raza criollo colombiano. La catalasa se encuentra en grandes cantidades en todas las fracciones de un eyaculado a excepción del fluido pre-espermático (38). Otras moléculas presentes en el plasma seminal, como vitamina $\mathrm{C}$, vitamina $\mathrm{E}$, urato, albumina, taurina, hipotaurina, piruvato y lactato, podrían actuar como antioxidantes $(15,16)$.

El mantenimiento de menores niveles de peroxidación lipídica de los espermatozoides, a pesar del evidente aumento en la producción de ERO en el semen congelado bajo suplementación con plasma seminal, podría entenderse como un efecto contrario al esperado. Sin embargo, estudios mostraron que el incremento de la generación de ERO no está necesariamente acompañada de un incremento detectable en la peroxidación lipídica (39). Adicionalmente, la medición no diferencial entre los tipos existentes de ERO, a través de la metodología empleada (FDA); así como el desconocimiento de los posibles mecanismos enzimáticos y mediados por 
moléculas de bajo peso molecular, involucrados en la desintoxicación de las mismas, no permiten dilucidar los eventos específicos relacionados con el control de la peroxidación. Entre los hallazgos más relevantes en este sentido está la evidente participación del $\mathrm{H}_{2} \mathrm{O}_{2}$ como la principal ROS causante de citotoxicidad en el semen equino (7); la reducción de alteraciones citológicas por acción de la catalasa y el glutatión reducido (39), y la disminución del estrés oxidativo y el mejoramiento de la calidad del semen refrigerado por efecto de la SOD (12).

Las correlaciones calculadas (Tabla 2) mostraron una asociación positiva entre la producción de ERO y la peroxidación lipídica, así como una asociación negativa entre estas variables, con los diferentes parámetros evaluados de fertilidad potencial, lo cual es indicador del efecto contraproducente de las ERO sobre la integridad y la funcionalidad de las células espermáticas. Las correlaciones positivas encontradas entre los parámetros: movilidad progresiva, vitalidad e integridad de membrana (HOS), demostraron la alta asociación existente entre estos indicadores de fertilidad potencial.

Se concluye que la suplementación del diluyente para la criopreservación de semen equino con plasma seminal, incrementa la producción de ERO y reduce la peroxidación lipídica del semen equino post-descongelación. Son necesarios estudios más detallados de los diferentes tipos de especies reactivas y de moléculas antioxidantes involucradas en los procesos de criopreservación de semen equino, con el fin de dilucidar sus efectos en la alteración o protección de las estructuras espermáticas al estrés oxidativo.

\section{Agradecimientos}

Dirección de investigaciones Politécnico Colombiano Jaime Isaza Cadavid. Laboratorio de Ciencia de los Alimentos, Universidad Nacional de Colombia, Sede Medellín.

\section{REFERENCIAS}

1. Ball, B. Oxidative stress, osmotic stress and apoptosis: Impacts on sperm function and preservation in the horse. Anim Reprod Sci 2008; 107:257-267.

2. Squires E, Keith S, Graham J. Evaluation of alternative cryoprotectants for preserving stallion spermatozoa. Theriogenology 2004; 62:1056-1065.

3. Mantovani R, Rota A, Falomo M, Bailoni L, Vincenti L. Comparison between glycerol and ethylene glycol for the cryopreservation of equine spermatozoa: semen quality assessment with standard analyses and with the hypoosmotic swelling test. Reprod Nutr Dev 2002; 42:217-226.

4. Pesch S, Bostedt $H$, Failing $K$, Bergmann M. Advanced fertility diagnosis in stallion semen using transmission electron microscopy. Anim Reprod Sci 2006; 91:285-298.

5. Pommer A, Rutllant J, Meyers S. The role of osmotic resistance on equine spermatozoal function. Theriogenology 2002; 58(7):13731384.
6. Devireddy R, Swanlund D, Olin T, Vincente $W$, Troedsson M, Bischof J et al. Cryopreservation of equine sperm: optimal cooling rates in the presence and absence of cryoprotective agents determined using differential scanning calorimetry. Biol Reprod 2002; 66:222-231.

7. Burnaugh L, Sabeur K, Ball B. Generation of superoxide anion by equine spermatozoa as detected by dihydroethidium. Theriogenology 2007; 67:580-589.

8. Kankofer M, Kolm G, Aurich J, Aurich C. Activity of glutathione peroxidase, superoxide dismutase and catalase and lipid peroxidation intensity in stallion semen during storage at $5^{\circ} \mathrm{C}$. Theriogenology 2005; 63:1354-1365.

9. Neild D, Gadella B, Colendrander B, Agüero A, Brouwers J. lipid peroxidation in stallion spermatozoa. Theriogenology 2002; 58:295-298.

10. Isachenko $E$, Isachenko $V$, Katkov $I$, Rahimi $G$, Schoèndorf $E$, Allmann $P$ et al. DNA integrity and motility of human spermatozoa after standard slow freezing versus cryoprotectant-free vitrification. Hum Reprod 2004; 19(44):932-939. 
11. Isachenko $\mathrm{V}$, Isachenko $\mathrm{E}$, Katkov I, Montag M, Dessole S, Nawroth F. Cryoprotectant-free cryopreservation of human spermatozoa by vitrification and freezing in vapor: effect on motility, DNA integrity, and fertilization ability. Biol Reprod 2004; 71:1167-1173.

12. Cocchia N, Pasolinia M, Mancini R, Petrazzuolo O, Cristofaro I, Rosapane I et al. Effect of sod (superoxide dismutase) protein supplementation in semen extenders on motility, viability, acrosome status and ERK (extracellular signal-regulated kinase) protein phosphorylation of chilled stallion spermatozoa. Theriogenology 2011; 75: 1201-1210.

13. Baumber J, Ball B, Linfor J. Assessment of the cryopreservation of equine spermatozoa in the presence of enzyme scavengers and antioxidants. Am J Vet Res 2005; 66(5):772-779.

14. Baumber J, Ball B. Determination of glutathione peroxidase and superoxide dismutase-like activities in equine spermatozoa, seminal plasma, and reproductive tissues. Am J Vet Res 2005; 66:1415-1419.

15. Ball B, Gravance C, Medina V, Baumber J. Liu I. Catalase activity in equine semen. Am J Vet Res 2000; 61(9):1026-1030.

16. Baumber J, Ball B, Gravance C, Medina V, Davies-Morel $M$. The effect of reactive oxygen species on equine sperm motility, viability, acrosomal integrity, mitochondrial membrane potential and membrane lipid peroxidation. J Androl 2000; 21(6):895-902.

17. Brito L, Greene L, Kelleman A, Knobbe M, Turner R. Effect of method and clinician on stallion sperm morphology evaluation. Theriogenology 2011; 76:745-750.

18. Graham J, Mocé E. Fertility evaluation of frozen/thawed semen. Theriogenology 2005; 64:492-504.

19. Neild D, Chaves G, Flores M, Mora N, Beconi $M$, Agüero A. Hypoosmotic test in equine spermatozoa. Theriogenology 1999; 51:721-727.

20. Henry M, Snoeck P, Cottorello A. Postthaw spermatozoa plasma integrity and motility of stallion semen frozen with different cryoprotectants. Theriogenology 2002; 58:245:248.
21. Perez-Osorio J, Mello F, Juliani G, Lagares M, Lago L, Henry, M. Effect on post-thaw viability of equine sperm using stepwise addition of dimethyl formamide and varying cooling and freezing procedures. Anim Reprod 2008; 5(3/4):103-109.

22. Bustamante I, Pederzolli C, Sgaravatti A, Gregory R, Dutra C, Jobim M etal. Skim milkegg yolk based semen extender compensates for non-enzymatic antioxidant activity loss during equine semen cryopreservation. Anim Reprod 2009; 6(2):392-399.

23. Medeiros A, Gomes G, Carmo M, Papa F, Alvarenga $M$. Cryopreservation of stallion sperm using different amides. Theriogenology 2002; 58:273-276.

24. Guthrie $H$, Welch $G$. Determination of intracellular reactive oxygen species and high mitochondrial membrane potential in Percolltreated viable boar sperm using fluorescenceactivated flow cytometry. J Anim Sci 2006; 84:2089-2100.

25. Morte $M$, Rodríguez $A$, Soares $D$, Rodrigues A, Gamboa S, Ramalho-Santos J. The quantification of lipid and protein oxidation in stallion spermatozoa and seminal plasma: Seasonal distinctions and correlations with DNA strand breaks, classical seminal parameters and stallion fertility. Anim Reprod Sci 2008; 106(1-2):36-47.

26. Salazar J, Teague S, Love C, Brinsko S, Blanchard T, Varner D. Effect of crypreservation protocol on postthaw characteristics of stallion sperm. Theriogenology $2011 ; 76: 409-418$.

27. Moore A, Squires E, Graham J. Effect of seminal plasma on the cryopreservation of equine. Theriogenologyy 2005; 63:2372-2381.

28. Lozano Benito D, Gil Huerta L, Álvarez San Martín C. Efecto de la adición de plasma seminal en el semen equino descongelado. Sanid mil 2011; 67(3):284-290.

29. Katila T, Anderson M, Reilas T, Koskinen E. Post-thaw motility and viability of fractionated and frozen stallion ejaculates. Theriogenology 2002; 58:241-244.

30. Alghamdi A, Foster D, Troedsson M. Equine seminal plasma reduces sperm binding to polymorphonuclear neutrophils (PMNs) and improves the fertility of fresh semen inseminated into inflamed uteri. Reproduc 2004; 127(5):593-600. 
31. Novak S, Smith T, Paradis F, Burwash L, Dyck $M$, Foxcroft G. Biomarkers of in vivo fertility in sperm and seminal plasma of fertile stallions. Theriogenology 2010; 74:956-967.

32. Pessanha M, Straggiotti J, Fagundes B, Costa J, De Souza G, Shimoya A. Efeito de proteínas do plasma seminal eqüino com massa superior a $10 \mathrm{kDa}$ concentradas 10 vezes sobre a congelabilidade do sêmen. R Bras Zootec 2008; 37(12):2115-2119.

33. Vasconcelos $A$, Santana $M$, Santos $A$, Santero M, Lagares M. Metabolic evaluation of cooled equine spermatozoa. Androl 2010; 42:106-111.

34. Weir C, Robaire B. Spermatozoa have decreased antioxidant enzymatic capacity and increased reactive oxygen species production during aging in the Brown Norway rat. J Androl 2007; 28:229-240.

35. Brum A, Sabeur K, Ball B. Apoptotic-like changes in equine spermatozoa separated by density-gradient centrifugation or after cryopreservation. Theriogenology 2008; 69:1041-1055.
36. Aitken RJ, Paterson $M$, Fisher $H$, Buckingham DW, Van Duin M. Redox regulation of tyrosine phosphorylation in human spermatozoa and its role in the control of human sperm function. J Cell Sci 1995; 108:2017-2025.

37. Villa N, Castaño $D$, Duque $P$, Ceballos $A$. Actividad de la glutatión peroxidasa y la superóxido dismutasa en sangre y plasma seminal en caballos colombianos. Rev Colomb Cienc Pecu 2012; 25:64-70.

38. Koskinen E, Karlsson M, Reilas T, Sankari S, Esala A, Katila T. Catalase activity and total protein in fractionated stallion seminal plasma. Theriogenology 2002; 58:337-340.

39. Baumber J, Ball B, Linfor J, Meyers S. Reactive oxygen species and cryopreservation promote DNA fragmentation in equine Spermatozoa. J Androl 2003; 24(4):621-628. 